\title{
Search for Leptoquarks and Compositeness at D0
}

\author{
Maxim Titov \\ Albert-Ludwigs University of Freiburg, Physics Institute, \\ Hermann-Herder Str.3, 79104, Freiburg, Germany \\ E-mail: maxim.titovephysik.uni-freiburg.de
}

\section{(on behalf of the D0 Collaboration)}

In this paper searches are presented for the pair production of first and second generation scalar leptoquarks and limits are given on the quark-lepton compositeness scale $\Lambda$ from protonantiproton collision data at a center-of-mass energy $1.96 \mathrm{TeV}$, collected with Run II D0 Detector in 2002-2004.

No evidence for a leptoquark signal has been observed in the topologies arising from $\mathrm{LQ}_{1} \mathrm{LQ}_{1} \rightarrow$ ejej and $\mathrm{LQ}_{1} \mathrm{LQ}_{1} \rightarrow$ ejvj, using an integrated luminosity of $252 \mathrm{pb}^{-1}$, and in the $\mathrm{LQ}_{2} \mathrm{LQ}_{2} \rightarrow \mu \mathrm{j} \mu \mathrm{j}$ channel, using a data sample corresponding to $294 \mathrm{pb}^{-1}$. From the upper bounds on the product of cross section times branching ratio $\beta=B r(L Q \rightarrow 1 j)$, a lower mass limits of $M_{L Q 1}$ $>241 \mathrm{GeV}$ and $\mathrm{M}_{\mathrm{LQ} 2}>247 \mathrm{GeV}$ for the first and second $\mathrm{LQ}$ generation are set for $\beta=1$. These results, combined with those obtained by D0 in Run I at a center-of-mass energy of $1.8 \mathrm{TeV}$, allow to exclude scalar LQ masses up to $256 \mathrm{GeV}$ and $251 \mathrm{GeV}$ (for $\beta=1$ ) for the first and second generation, respectively. These limits are the most stringent up-to-date constraints on the first and second generation LQ masses, which are independent of the strength of the leptonquark-leptoquark $\left(\lambda_{\mathrm{LQ}}\right)$ Yukawa coupling.

The dilepton mass spectra in $\mathrm{pp} \rightarrow 1^{+} 1^{-}+\mathrm{X}$ interactions are studied using dielectron (dimuon) data samples, corresponding to an integrated luminosity of $271 \mathrm{pb}^{-1}\left(406 \mathrm{pb}^{-1}\right)$. The mass spectra being a probe for new physics are examined for new interactions of quarks and leptons from a common composite structure. No excess of events is found over the expectation from Standard Model processes. The current experimental lower limits on the compositeness scale, $\Lambda$, vary, for different chirality channels, from 3.6 to $9.1 \mathrm{TeV}$ for the (eeqq) and from 4.2 to $9.8 \mathrm{TeV}$ for the ( $\mu \mu \mathrm{qq})$ contact interaction. The D0 Run II limits on ( $\mu \mu \mathrm{qq})$ are currently the most stringent bounds for the quark-muon compositeness scale.

European Physical Society

HEP2005 International Europhysics Conference on High Energy Physics

EPS (July 21st-27th 2005) in Lisboa, Portuga

\footnotetext{
${ }^{*}$ Speaker
} 


\section{Scalar Leptoquark Phenomenology}

Leptoquarks (LQ) are color-triplet bosons, carrying both baryon and lepton quantum numbers and fractional electric charge. They appear in many extensions beyond the standard model (SM) such as composite models with quark and lepton substructure, extended technicolor, SUSY with R-parity violations and grand unified theories (GUT), based on gauge groups $\mathrm{SU}(5), \mathrm{SO}(10)$ with Pati-Salam SU(4) color symmetry, $\mathrm{SU}(15)$ and superstring-inspired E6 models [1]. The general classification of leptoquark states, proposed by Buchmuller, Ruckl and Wyler, contains 7 scalar and 7 vector leptoquarks [2]. At the Tevatron, scalar leptoquarks would be pair produced dominantly through quark-antiquark annihilation (for $\mathrm{M}_{\mathrm{LQ}}>100 \mathrm{GeV}$ ) and gluon fusion [3]. Since the gluon-leptoquark interactions are determined by the non-Abelian $\mathrm{SU}(3)_{\mathrm{C}}$ gauge symmetry of scalar QCD, the cross section for scalar LQ pair production is essentially independent of the lepton-quark-LQ Yukawa coupling $\left(\lambda_{\mathrm{LQ}}\right)$, which contributes only $\sim 1 \%$ (for $\lambda_{\mathrm{LQ}} \sim \lambda_{\mathrm{em}}$ ) of the total cross section in t-channel lepton exchange of qq-annihillation. The cross section coincides with those of squark-pair production in the limit of large gluino masses [3]. While the scalar LQ pair production at the Tevatron does not depend on its electroweak properties, leptoquarks are generally assumed to have a family diagonal coupling (LQ couples to a single lepton/quark generation only - to avoid flavor-changing neutral currents (FCNC) or lepton flavor violation) and chiral coupling (to obey bounds arising from atomic parity violation and to avoid anomalously large contributions to $\pi \rightarrow \mathrm{ev}$ decays). Finally, LQ couplings to fermions are assumed to be baryon and lepton number conserving, to avoid rapid proton decays.

\section{First and Second Generation Scalar Leptoquarks Limits}

The experimental signatures for the LQ pair production at the Tevatron are two jets accompanied by either two leptons (ljlj), lepton and missing transverse energy $\mathrm{E}_{\mathrm{T}}{ }^{\mathrm{miss}}$ (lvjj), or $\mathrm{E}_{\mathrm{T}}{ }^{\text {miss }}(v v \mathrm{jj})$, with each pair of jet +lepton invariant masses being equal to the leptoquark mass.

D0 has searched for the first generation scalar leptoquarks in the $\mathrm{LQ}_{1} \mathrm{LQ}_{1} \rightarrow$ ejej and ejvj channels based on Run II integrated luminosity of $252 \mathrm{pb}^{-1}$. The (ejej) analysis requires two electrons with transverse energy $\mathrm{E}_{\mathrm{T}}^{\mathrm{e}}>25 \mathrm{GeV}$ and at least two jets with $\mathrm{E}_{\mathrm{T}}^{\mathrm{j}}>20 \mathrm{GeV}$. The (evjj) data sample is selected with one electron $\left(\mathrm{E}_{\mathrm{T}}^{\mathrm{e}}>35 \mathrm{GeV}\right)$, more than two jets with $\mathrm{E}_{\mathrm{T}}^{\mathrm{j}}>25 \mathrm{GeV}$, and $\mathrm{E}_{\mathrm{T}}^{\text {miss }}>30 \mathrm{GeV}$. The major background that mimic (ejej) decay of LQ pair are $\mathrm{Z} / \gamma^{*}+2$ jets, $\mathrm{tt}$ production and misidentified multi-jet events, while the primary backgrounds to the (evjj) final state are $\mathrm{W}+2$ jets, multijet and tt production. A significant discrimination between the LQ signal and the major $\mathrm{Z} / \gamma^{*}+$ jets and $\mathrm{W}+$ jets backgrounds is achieved by a cut on the scalar transverse energy sum: $\mathrm{S}_{\mathrm{T}}=\mathrm{E}_{\mathrm{T}}{ }^{\mathrm{j} 1}+\mathrm{E}_{\mathrm{T}}{ }^{\mathrm{j} 2}+\mathrm{E}_{\mathrm{T}}{ }^{\mathrm{e} 1}+\mathrm{E}_{\mathrm{T}}{ }^{\mathrm{e} 2}>450 \mathrm{GeV}$ for the (ejej) and $\mathrm{S}_{\mathrm{T}}=\mathrm{E}_{\mathrm{T}}{ }^{\mathrm{j} 1}+\mathrm{E}_{\mathrm{T}}{ }^{\mathrm{j} 2}+$ $\mathrm{E}_{\mathrm{T}}{ }^{\mathrm{e} 1}+\mathrm{E}_{\mathrm{T}}{ }^{\text {miss }}>330 \mathrm{GeV}$ for the (evej) final state. As no excess of data above background is found after the final $\mathrm{S}_{\mathrm{T}}$ cut, a $95 \%$ C.L. limit on the first-generation $\mathrm{LQ}$ mass is set at $\mathrm{M}_{\mathrm{LQ1}}>241 \mathrm{GeV}$ $\left(\mathrm{M}_{\mathrm{LQ1}}>218 \mathrm{GeV}\right)$ for $\beta=\operatorname{Br}(\mathrm{LQ} \rightarrow \mathrm{lj})=1(\beta=1 / 2)$, based on Run II combination of (ejej) and (evjj) channels, using a Bayesian likelihood technique [4]. The D0 Run II and combined Run I+II lower limits, on the first-generation leptoquark mass as a function of $\beta$, are shown in Fig.1 [5]. 
D0 has also performed a similar search for second generation scalar $\mathrm{LQ}_{2}$ decaying through $\mathrm{LQ}_{2} \mathrm{LQ}_{2} \rightarrow \mu \mathrm{j} \mu \mathrm{j}$. The Run II integrated luminosity of the data used in this analysis is $294 \mathrm{pb}^{-1}$. After the initial event selection, which requires the presence of two muons with $\mathrm{p}_{\mathrm{T}}>15 \mathrm{GeV}$ and two jets with $\mathrm{E}_{\mathrm{T}}^{\mathrm{j}}>25 \mathrm{GeV}$, the background is dominated by the $\mathrm{Z} / \gamma^{*}+\mathrm{jets}$ and $\mathrm{tt}$ production. The $\mathrm{LQ}_{2} \mathrm{LQ}_{2} \rightarrow \mu \mathrm{j} \mu \mathrm{j}$ events are expected to have both high di-muon masses $\left(\mathrm{M}_{\mu \mu}\right)$ and large values of $\mathrm{S}_{\mathrm{T}}=\mathrm{E}_{\mathrm{T}}{ }^{\mathrm{j} 1}+\mathrm{E}_{\mathrm{T}}{ }^{\mathrm{j} 2}+\mathrm{p}_{\mathrm{T}}{ }^{\mu 1}+\mathrm{p}_{\mathrm{T}}{ }^{\mu 2}$. Therefore, the sensitivity to $\mathrm{LQ}$ decays is studied using the twodimensional distribution $\left(S_{T}\right.$ versus $\left.M_{\mu \mu}\right)$ divided into four signal bins, in order of increasing signal over background ratio (S/B). No excess of data over SM background was found. The D0 Run II lower limit at the $95 \%$ C.L., calculated by treating the four signal bins as individual channels and combining them using the modified frequentist approach [6], on the mass of the second generation scalar leptoquark is set at $\mathrm{M}_{\mathrm{LQ} 2}>247 \mathrm{GeV}\left(\mathrm{M}_{\mathrm{LQ} 2}>182 \mathrm{GeV}\right)$ for $\beta=1(\beta=1 / 2)$, respectively. The combined D0 Run I+II lower limit for a combination of channels $(\mu \mathrm{j} \mu \mathrm{j})$ and ( $v j \mu \mathrm{j})$ ( $\mathrm{vj \mu j}$ channel was analyzed only at Run I) for the second-generation scalar LQ is $\mathrm{M}_{\mathrm{LQ} 2}>251 \mathrm{GeV}\left(\mathrm{M}_{\mathrm{LQ} 2}>204 \mathrm{GeV}\right)$ for $\beta=1(\beta=1 / 2)$. The excluded parameter regions, which are independent from $\lambda_{\mathrm{LQ}}$, are shown in Fig.1 (right).
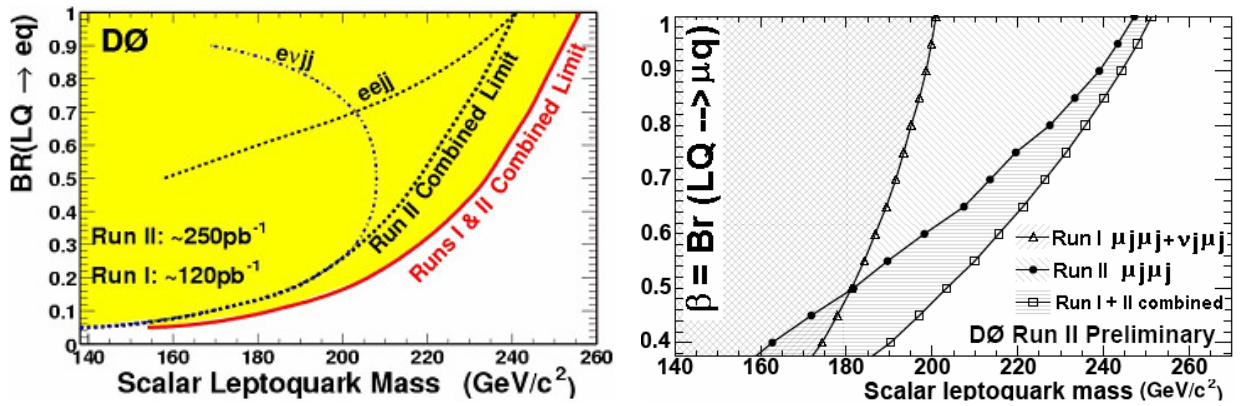

Fig. 1 The D0 Run II and combined Run I+II excluded regions (filled/shaded area) in the $\beta=\mathrm{Br}$ (LQ $\rightarrow \mathrm{lj}$ ) versus LQ mass plane for the first-generation (left) and second-generation (right) scalar leptoquarks.

\section{Contact Interactions and Compositeness Limits}

A general framework to assess new physics, appearing from the interference of any new particle field, associated to a characteristic energy scale $\left(\Lambda^{2}>>\mathbf{s}\right)$, with the $\gamma$ and $Z$ fields of the $\mathrm{SM}$, is a concept of four-fermion point-like (ff)(ff) contact interactions (CI) [7]. The most general Lagrangian for CI between leptons and quarks can be written in the form:

$$
\mathrm{L}=\Sigma_{\mathrm{q}} \Sigma_{\mathrm{A}, \mathrm{B}} \eta_{\mathrm{AB}}{ }^{\mathrm{q}}\left(1_{\mathrm{A}} \gamma_{\mu} 1_{\mathrm{A}}\right)\left(\mathrm{q}_{\mathrm{B}} \gamma_{\mu} \mathrm{q}_{\mathrm{B}}\right) ; \quad \eta_{\mathrm{AB}}{ }^{\mathrm{q}}=\varepsilon_{\mathrm{AB}}\left(\mathrm{g} / \Lambda_{\mathrm{AB}}{ }^{\mathrm{q}}\right)^{2}
$$

where $\eta_{\mathrm{AB}}$ describes the chiral structure of the interaction $(\mathrm{A}, \mathrm{B}=\mathrm{L}, \mathrm{R})$, and the $\Sigma_{\mathrm{q}}$ extends over all up-type and down-type quarks and antiquarks, $g / \Lambda$ is an effective coupling over the scale $\Lambda$, $\varepsilon_{\mathrm{AB}}=-1(+1)$ defines constructive (destructive) interference of the CI with the SM. Any new phenomena, such as compositeness $(\Lambda \sim$ scale of composite objects $)$, or the exchange of heavy leptoquarks $\left(\mathrm{g} / \Lambda \sim \lambda_{\mathrm{LQ}} / \mathrm{M}_{\mathrm{LQ}}\right)$, can be described by an appropriate choice of $\eta_{\mathrm{AB}}$. Compositeness models postulate common constituents of the SM fermions and a new strong dynamics ('metacolor') that bind these constituents. Experimentally, quark compositeness is the most sensitive to the deviation in production of high-transverse momentum jets relative to SM 
predictions. Quark-lepton compositeness would modify the SM Drell-Yan cross section for lepton pair production in the high mass region above the $\mathrm{Z}$ peak.

The $\mathrm{Z} / \gamma^{*} \rightarrow$ ee $\left(\mathrm{Z} / \gamma^{*} \rightarrow \mu \mu\right)$ invariant mass spectra from $271 \mathrm{pb}^{-1}\left(406 \mathrm{pb}^{-1}\right)$ of collisions, taken by the D0 detector in Run II, are used to measure the shape of the cross sections and to search for continuum anomalies with respect to the SM predictions. To avoid biases from backgrounds, the data selection is optimized for purity, by requiring two electrons of $\mathrm{p}_{\mathrm{T}}^{\mathrm{e}}>25$ $\mathrm{GeV}$ in the dielectron, and two isolated muons with $\mathrm{p}_{\mathrm{T}}{ }^{\mu}>15 \mathrm{GeV}$ and $\mathrm{M}_{\mu \mu}>50 \mathrm{GeV}$ in the dimuon analysis. The major backgrounds for $\mathrm{Z} / \gamma^{*} \rightarrow$ ee - QCD multijet and $\gamma+$ jet events, where both objects fake electrons, are determined directly from the data. No evidence for physics beyond the SM is observed both in $\mathrm{e}^{+} \mathrm{e}^{-}$and $\mu^{+} \mu^{-}$data. The lower limits on the quark-lepton compositeness scale are set independently for each chirality channel of the CI Lagrangian, using a Bayesian analysis of the shape of the $M_{e e}$ mass distributions of events or from the twodimensional distributions $\left(\mathrm{M}_{\mu \mu}\right.$ vs. $\left.\cos \theta^{*}\right)$. The D0 Run II lower bounds on the quark-electron and quark-muon compositeness scale $\Lambda$ range from 3.6 to $9.1 \mathrm{TeV}$ for (eeqq) and from 4.2 to 9.8 TeV for ( $\mu \mu \mathrm{qq}$ ) CI (see Fig. 2). The current summary of 95\% C.L. lower limits on the CI energy scale $\Lambda$ from LEP, HERA and Tevatron collider experiments is also shown in Fig.2.

\begin{tabular}{|c|r|r|r|r|}
\hline Model & \multicolumn{2}{|c|}{$\Lambda-(\mathrm{TeV})$} & \multicolumn{2}{c|}{$\Lambda+(\mathrm{TeV})$} \\
\hline & $\mathrm{ee}$ & $\boldsymbol{\mu} \mu$ & $\mathrm{ee}$ & $\boldsymbol{\mu} \mu$ \\
\hline LL & 6.2 & 7.0 & 3.6 & 4.2 \\
\hline RR & 5.8 & 6.7 & 3.8 & 4.2 \\
\hline LR & 4.8 & 5.1 & 4.5 & 5.3 \\
\hline RL & 5.0 & 5.2 & 4.3 & 5.3 \\
\hline LL+RR & 7.9 & 9.0 & 4.1 & 5.0 \\
\hline LR+RL & 6.0 & 6.1 & 5.0 & 6.4 \\
\hline LL-LR & 6.4 & 7.7 & 4.8 & 4.9 \\
\hline RL-RR & 4.7 & 7.4 & 6.8 & 5.1 \\
\hline VV & 9.1 & 9.8 & 4.9 & 6.9 \\
\hline AA & 7.8 & 9.8 & 5.7 & 5.5 \\
\hline
\end{tabular}

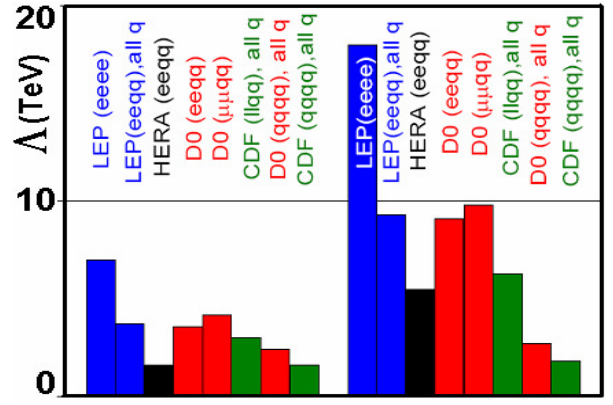

Fig. 2. (Left) The 95\% C.L. D0 Run II lower limits on the quark-electron and quark-muon compositeness scale for constructive $\left(\Lambda^{-}\right)$and destructive $\left(\Lambda^{+}\right)$CI interference with the SM Lagrangian. (Right) Summary of the $95 \%$ C.L. lower limits on the electron (eeee), quark (qqqq) and quark-lepton (llqq) compositeness scale $\Lambda$ of contact interactions from LEP [8], HERA [9] and Fermilab (CDF[10], D0) Experiments. The lower and upper values of $\Lambda$ range, among the different chirality channels, are shown. (For LEP, (eeqq) CI are assumed to couple to all quark flavors with equal strength. The CDF Run I (llqq) limits assume that leptons couple symmetrically to u-type $(\mathrm{u}, \mathrm{c}, \mathrm{t})$ and d-type $(\mathrm{d}, \mathrm{s}, \mathrm{b})$ quarks. All quarks families are assumed to be composite for (qqqq) CI).

\section{References}

[1] D. Acosta, S. Blessing, Ann. Rev. Nucl. Part. Sci 49 (389-434), 1999.

[2] W. Buchmuller, R.Ruckl, D.Wyler, Phys. Lett. B. 191(4) (442-448), 1987.

[3] M. Kramer et al., Phys. Rev. Lett. 79(3) (341-344), 1997.

[4] C. Pilcher, G. Landsberg, M. Paterno, [hep-ex/9810015].

[5] V. Abazov et al., Phys. Rev. D. 71, 071104, 2005; http://www-d0.fnal.gov/Run2Physics/WWW/results/np.htm

[6] T. Junk, Nucl. Instrum .Methods A. 434 (435), 1999.

[7] E. Sichten, K. Lane, M. Peskin, Phys.Rev. Lett. 50(11) (811-814), 1983.

[8] LEPEWWG ff Subgroup, http://lepewwg.web.cern.ch/LEPEWWG/lep2

[9] C. Adloff et al., Phys. Lett. B. 568 (35-47), 2003.

[10] F. Abe et al., Phys. Rev. Lett. 79(12) (2198-2203), 1997. 